\title{
THE X-RAY SPECTRUM OF SUPERNOVA SN1993J
}

its Long-Term Evolutions, and Shock Heating.

SHIN'ICHIRO UNO, KAZUHISA MITSUDA, TADAYUKI TAKAHASHI, HAJIME INOUE AND FUMIYOSHI MAKINO

Institute of Space and Astronautical Science

3-1-1 Yoshino-dai Sagamihara-city Kanagawa 229 Japan

KAZUO MAKISHIMA

Department of Physics, the University of Tokyo

Bunkyo-ku Tokyo 113 Japan

YOSHITAKA ISHISAKI

Dept. of Physics, Tokyo Metropolitan University

Minami-Osawa 1-1, Hachioji, Tokyo 192-03, Japan

YOSHIKI KOHMURA

The Institute of Chemical and Physical Research

3-1, Hirosawa, Wako, Saitama 351-01 Japan

MASAYUKI ITOH

Faculty of Human Development, Kobe University

3-11 Tsurukabuto Nada-ku Kobe 657

AND

WALTER .H.G. LEWIN

Massachusetts Institute of Technology

Center for Space Research, Room 37-627, Cambridge, MA 02139

SN1993J is very unique object which was discovered in the nearby $\mathrm{Sb}$ galaxy M81 (NGC 3031) on March 28[1]. The first detection of the radio emission was at $22.5 \mathrm{GHz}$ by the VLA only 5 days after the optical outburst[2]. Subsequently X-ray emission was detected by ROSAT and ASCA at 6 days and 8 days after the explosion respectively. These emissions are expected when the SN shock front sweeps out the circumstellar matter (CSM). The early detection of radio and X-ray emission implies the existence of high-density CSM in the vicinity of the supernova(e.g. [3][4]).

ASCA[5] observed SN1993J eleven times during 1993 April to 1995 October. Although the two bright X-ray sources, M81 X-5 and X-6 are only about 3 arc minutes and 1 arc minute from the supernova, we successfully 
separated the three sources for the SIS (Solidstate Imaging Spectometer) data utilizing a kind of 2-dimensional image fittings and obtained the spectra of the three sources.

The spectrum showed drastic softening with a power-law photon index of 0.4 to 4 , while the X-ray intensity decreased from 0.03 to 0.008 counts/sec/SIS. The early-phase of spectra require two thermal emission components of different absorption columns, if they are fitted with thermal models. The temperatures of two emission components cannot be well constrained from ASCA contiuum spectra. However, the detection of iron $\mathrm{K}$ emission line with ASCA [4] and the hard X-ray spectra observed by OSSE imposes strong constraints on the temperatures.

The properties of the two emission components are consistent with those of emissions from the front and reverse shocks of the supernova explosion. The drastic softening of the X-ray spectra is explained by decrease of the absorption column density of the initially heavily- abosrbed reverse shock component; the dominant emission component in the soft X-ray band altered from the front shock to the revserse shock.

We investigated the emission line structure in detail. The central energy of the line is $6.70 \pm 0.15 \mathrm{keV}$, and width of $0.4 \pm 0.2$ for the spectrum of 10 days after the explosion. The interpretation of the center energy and the line width involves, the geometry and the motion of the supernova shock.

The continuum X-ray spectra could be described with the two-shock scheme. In such models, the emission line is estimated to come from "reverse shock" and "cold shell". Considering bloadning and blue-shift by the expansion veolocity of the supernova, we fitted the emission line profile with three gaussian models, i.e. Hydrogen-like, Helium-like and neutral Iron. From the ratio of the line equivalent widths of 6.9 to $6.7 \mathrm{keV}$ reflects the temperature of the plasma. The temperature on April 5 to be between 3 $\mathrm{keV}$ and $20 \mathrm{keV}$ and the temperature of April 7 and April 16 to be lower than $12 \mathrm{keV}$. This result is consistent with the two shock scheme.

1. Garcia, F. et al. 1993, IAUC, 5731

2. Weiler, K. W. et al. 1993, IAUC, 5752

3. Zimmermann, H. U., et al. 1994, nature, 367, 621-623

4. Kohmura, Y., et al., 1994, Publ. Astron. Soc. Japan, 46, L157-L161

5. Tanaka, Y., et al., 1994, Publ. Astron. Soc. Japan, 46, 37

6. Leising, M. D. et al., 1994, Astrophys. J. Let.,431, 95

7. Chevalier, R. A. 1982, Astrophys. J. ,259, 302-310 